\title{
Editorial: Evolving Virtual and Computational Paleontology
}

\author{
Luca Pandolfi ${ }^{1 * t}$, Pasquale Raia ${ }^{2 \dagger}$, Josep Fortuny ${ }^{3 \dagger}$ and Lorenzo Rook ${ }^{1 \dagger}$ \\ ${ }^{1}$ Department of Earth Sciences, University of Florence, Florence, Italy, ${ }^{2}$ Department of Earth Sciences, Environmental and \\ Resources, University of Naples "Federico II," Naples, Italy, ${ }^{3}$ Institut Català de Paleontologia Miquel Crusafont, Cerdanyola del \\ Vallès, Spain
}

Keywords: digital models, surface scanning, photogrammetry, computed tomography, computational analyses

Editorial on the Research Topic

Evolving Virtual and Computational Paleontology

During the last few decades, the development of new technologies and methods, and increases in computational power, are widening Paleontology's research frontiers, moving this discipline-situated halfway between Biology and Geology-toward increasing use of (paleo) biological and (paleo)ecological approaches, and providing the opportunity to fully explore quantitative aspects of phenotypic evolution as well as new clues to the history of life. The everincreasing availability of 3D models obtained by CT, surface scanning (SC) and/or photogrammetry (PT) is increasing the visibility and accessibility of rare, difficult to handle, remains, and therefore the number of virtual fossil representations that feature in scientific papers, contributing to this "revolution" and leading to the dawn of the "Virtual and Computational Paleontology" era. The pioneering steps toward this new era were taken in the early 1980s, when Tate and Cann (1982) and Conroy and Vannier (1984) became the first research teams to apply CT to Vertebrate Paleontology. Almost two decades later, the first applications of SC and PT to Vertebrate Paleontology opened the door to many new ideas and approaches (Lyons et al., 2000; Breithaupt and Matthews, 2001). Since the beginning of this digital paleontological era, the number of scientists merging traditional techniques with virtualization and computational advances has greatly increased, a process that still continues (and the first comprehensive reviews were published a few years later - see Zollikofer and Ponce de Leon, 2005; Cardini and Loy, 2013; Sutton et al., 2014).

Virtual (digital) representations have a great variety of non-invasive applications, including but not limited to digital curation (based on 3D models libraries), virtual restoration of specimens, and anatomical studies of both external and internal morphological structures, and open up the opportunities for development of new analytical tools. Thanks to virtualization, many Computational analyses and techniques (including geometric morphometrics, finite element analysis, multibody dynamic analysis, computational fluid dynamics, machine-learning, etc.) have been widely applied in Vertebrate Paleontology. Furthermore, their use is currently rapidly increasing thanks to continuing efforts to create more and better $3 \mathrm{D}$ virtual models, which are the basis for new computational approaches. Despite these many benefits, Virtual and Computational Paleontology is still not prevalent as it might be. Virtual data are therefore not broadly shared and new methodologies and techniques usually do not easily reach the widest audience, in particular younger researchers who usually show an insatiable hunger for learning and acquiring new skills.

This Research Topic was conceived with the principal goal of spreading this technological knowledge and, by taking advantage of the Open Access format of Frontiers, was intended to reach the widest possible number of researchers. The volume includes 15 different papers on the use and analysis of virtual representations and new computational methods, applied to several vertebrate taxa. 
3D models of external structures, obtained by means of SC and PT were the study subjects of several papers. Bartolini Lucenti et al. used a $3 \mathrm{D}$ visualization and digital imaging technique to study a new fossil canid from Georgia. They applied for the first time augmented reality to a few specimens through a simple web app. A new virtual reconstruction protocol, called Target Deformation, was applied by Cirilli et al., to $3 \mathrm{D}$ models of fossil horse skulls. This method allowed to virtually restore the strongly deformed holotype of Equus stenonis by using undeformed skull fragments of the same species found elsewhere. A caudal series of the dinosaur Giraffatitan was digitalized by Díez Díaz et al. via PT. The authors presented the first detailed 3D volumetric reconstruction of the caudal epaxial and hypaxial musculature of this sauropod. PT was at the core of Mujal et al.'s study, applied to Permian and Triassic tetrapod footprints. They used their 3D data to not only describe footprints but also to offer an initial qualitative interpretation of relative depth patterns within the tracks and their functional implications. Serio et al. combined PT and geometric morphometrics to study morphological convergence in the humerus of Xenarthra. The authors suggested that the highly specialized morphologies seen in digging taxa and tree sloths reflect major deviations from the plesiomorphic xenarthran body plan which occurred several times during the history of the group. Morphological convergence was the issue with Sansalone et al. who investigated the humeri of fossorial mammals by means of CT scans. They showed that fossorial mammals evolved multiple strategies to exploit the subterranean ecotope, characterized by different functional trade-offs rather than by convergence toward a single adaptive optimum.

Data on internal structures were obtained by means of CT scans of several fossil taxa, and 3D models and images were analyzed using several techniques. In Boscaini et al. endocranial features of giant sloths revealed by CT, helps elucidating the evolutionary history of sloths, whereas Iurino et al. described the first brain endocast of a juvenile fossil rhinoceros obtained via the CT method. Melchionna et al. used information on brain size and asymmetry in Homo species to argue that the emergence of handedness and early manifestations of cultural modernity nicely coincided with the emergence of the three species with the largest and most rapidly evolving brains among hominoids, namely Homo heidelbergensis, Homo neanderthalensis and Homo sapiens.

The variations in shape of stapes of Ruminantia was explored by Mennecart et al. on 3D tomographic data, whereas Pusch et al. reported endocranial data from Permo-Triassic therapsids,

\section{REFERENCES}

Breithaupt, B. H., and Matthews, N. A. (2001). "Preserving paleontological resources using photogrammetry and geographic information systems," in Crossing boundaries in park management: proceedings of the 11th conference on research and resource management in parks and public lands, Denver, CO, April 16-20, 2001. Editor D. Harmon (Hancock, MI: The George Wright Society, Inc.), 62-70.

Cardini, A., and Loy, A. (2013). Virtual morphology and evolutionary morphometrics in the new millenium. Hystrix 24 (1), 1-5. doi:10.4404/ hystrix-24.1-8749 redescribing the skull of the therocephalian Lycosuchus on the basis of CT reconstruction. Püschel et al. studied a fossil primate from Cuba, Paralouatta, using 3D talar models and geometric morphometrics combined with machine-learning classification algorithms to argue for some degree of terrestriality in this extinct platyrrhine.

In addition, the Research Topic covers several other aspects of Virtual and Computational Paleontology, including methodological approaches and problems. Pérez Ramos and Figueirido focused on using virtual approaches to solve technical issues commonly encountered by paleontologists and evolutionary biologists. The authors proposed various solutions and used fossil skulls of the cave bears to test them. Piras et al. investigated visualization of local deformation in modern shape analysis, considering paleontological case studies and proposing new computational methods for evaluating and visualizing local deformations. Neutron microtomography was successfully used by Zanolli et al. to extract, three-dimensionally render and quantitatively assess the internal morphological details of fossil hominid material from different localities and strata; This Research Topic represents a new step in the development of methods for use of virtual representations of specimens by the scientific community. The Topic also explores how these models provide the basis for numerous applications, techniques and methodologies, providing new insights into vertebrate paleobiology. Finally, the Topic serves as both a meeting point for the new generation of paleontologists and evolutionary biologists, and an updated overview of Virtual and Computational Paleontology, a discipline that is rapidly evolving thanks to a never-ending stream of technological improvements.

\section{AUTHOR CONTRIBUTIONS}

All authors listed have made a substantial, direct, and intellectual contribution to the work, and approved it for publication.

\section{ACKNOWLEDGMENTS}

We would like to thank all reviewers for their contributions, and all authors of the submitted manuscripts. We also would like to thank the editor Corwin Sullivan and the Frontiers Team for their support and suggestions.

Conroy, G. C., and Vannier, M. W. (1984). Noninvasive three-dimensional computer imaging of matrix-filled fossil skulls by high-resolution computed tomography. Science 226 (4673), 456-458. doi:10.1126/science.226.4673.456

Lyons, P. D., Rioux, M., and Patterson, R. T. (2000). Application of a threedimensional color laser scanner to paleontology: an interactive model of a juvenile Tylosaurus sp. basisphenoid-basioccipital. Palaeontol. Electron. 3 (2), 4A.

Sutton, M. D., Rahman, I. A., and Garwood, R. J. (2014). Techniques for virtual palaeontology. London, UK: Wiley Blackwell, 208.

Tate, J. R., and Cann, C. E. (1982). High-resolution computed tomography for the comparative study of fossil and extant bone. Am. J. Phys. Anthropol. 58 (1) 67-73. doi:10.1002/ajpa.1330580108 
Zollikofer, C. P., and Ponce de Leon, M. (2005). Virtual reconstruction: a primer in computer-assisted paleontology and biomedicine. Hoboken, NJ: John Wiley and Sons, 333 .

Conflict of Interest: The authors declare that the research was conducted in the absence of any commercial or financial relationships that could be construed as a potential conflict of interest.
Copyright $\odot 2020$ Pandolfi, Raia, Fortuny and Rook. This is an open-access article distributed under the terms of the Creative Commons Attribution License (CC BY). The use, distribution or reproduction in other forums is permitted, provided the original author(s) and the copyright owner(s) are credited and that the original publication in this journal is cited, in accordance with accepted academic practice. No use, distribution or reproduction is permitted which does not comply with these terms. 